\title{
Erratum to: Parabolic Motion-Vector Re-estimation Algorithm for Compressed Video Downscaling
}

\author{
Chia-Hung Yeh • Ying-Hsiang Chen • Ming-Chieh Chi • \\ Mei-Juan Chen
}

Published online: 30 March 2010

(C) Springer Science+Business Media, LLC 2010

\section{Erratum to: J Sign Process Syst}

DOI 10.1007/s11265-010-0455-Z

The original version of this article unfortunately contained a mistake. The name of the second author was not presented correctly. "Ying H. Chen" should be "Ying-Hsiang Chen."

The online version of the original article can be found at http://dx.doi. org/10.1007/s11265-010-0455-z.

C.-H. Yeh $(\bowtie) \cdot$ M.-C. Chi

National Sun Yat-Sen University,

No. 70, Lien-hai Road, Kushan District,

Kaohsiung, 804 Taiwan, Republic of China

e-mail: yeh@mail.ee.nsysu.edu.tw

M.-C. Chi

e-mail: mingchieh.chi@gmail.com

\section{Y.-H. Chen}

National Health Research Institute,

No. 35, Keyan Road, Zhunan Town,

Miaoli County, 350 Taiwan, Republic of China

e-mail: chen.ying.hsiang@gmail.com

\section{M.-J. Chen}

National Dong-Hwa University,

1, Sec. 2, Da Hsueh Rd., Shou-Feng,

Hualien, 974 Taiwan, Republic of China

e-mail: cmj@mail.ndhu.edu.tw 